\title{
APPROACH TO THE HIP
}

\section{A Suggested Improvement on Kocher's Method}

\author{
Bryan McFarland and Geoffrey Osborne, Liverpool, England \\ From the Department of Orthopaedic Surgery, The University of Liverpool
}

This paper describes a method of preserving the integrity of the gluteus medius muscle during the posterior approach to the hip joint. The present considerable interest in this approach is largely due to Gibson's admirable description in 1950 of Kocher's incision, and also, rather coincidentally, to the general increased interest in arthroplasty of the hip. While the anterior Hueter incision is recommended by the Judet brothers, many surgeons find that more work can be carried out with the more ample exposure of the posterior route. The very mobility imparted by arthroplasty, however, demands preservation of gluteal power to a degree little short of normal-certainly to a degree not always provided by the Kocher and Gibson approaches, because transverse division of the gluteus medius or its trochanteric insertion does not always result in satisfactory abductor power, even after strong suture or bony fixation. It was with the purpose of preserving the total continuity of the muscle that the method here described was evolved.

\section{ANATOMY}

The procedure depends on the anatomical observation that the gluteus medius and vastus lateralis can be regarded as being in direct functional continuity through the thick " tendinous" periosteum covering the greater trochanter. Moreover, these two muscles meet at an angle, open anteriorly, so that by loosening the periosteum from the trochanter it is possible to displace forward the combined muscle bellies like a bucket handle. In practice, the periosteum may not always strip easily from the trochanter, and in order to preserve intact the tendinous junction it may be convenient to shave the bony surface of the trochanter so that a few thin flakes of bone adhere to the tendon.

\section{DETAILS OF TEGHNIQUE}

The approach is essentially lateral and gives a view of the anterior, posterior and superior aspects of the hip joint. The skin incision (Fig. l) is mid-lateral, centred over the greater trochanter, the tendinous junction of the gluteus maximus and tensor fasciae latae above, and the middle of the shaft of the femur below: its length depends on the amount of subcutaneous fat. The gluteal fascia and ilio-tibial band are exposed and divided in the straight mid-lateral line along the whole length of the skin incision (Fig. 2). The gluteus maximus is retracted posteriorly, and the tensor fasciae latae anteriorly. The gluteus medius is exposed and by blunt dissection is separated from the pyriformis and gluteus minimus deeply. The posterior border of the gluteus medius is clearly defined, and the point at which it joins the prominent posterior edge of the greater trochanter is identified. From this point an incision down to bone is made through the periosteum and tendinous fascia obliquely downwards, across the trochanter to the middle of the lateral aspect of the femur, and is continued downwards in the vastus lateralis to the lower limit of the original incision (Fig. 3). The attachment of gluteus medius to the trochanter, and the periosteum and the tendinous junction of medius and lateralis, and the origin of lateralis are then peeled off the bone in one piece. This must be done with a scalpel or with a sharp chisel: it is not usually possible to do 


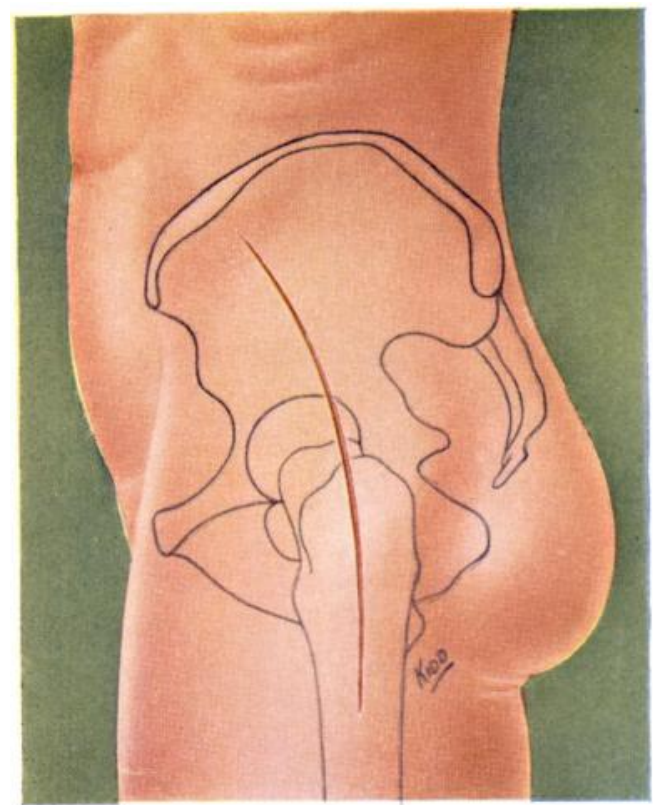

Fili. 1

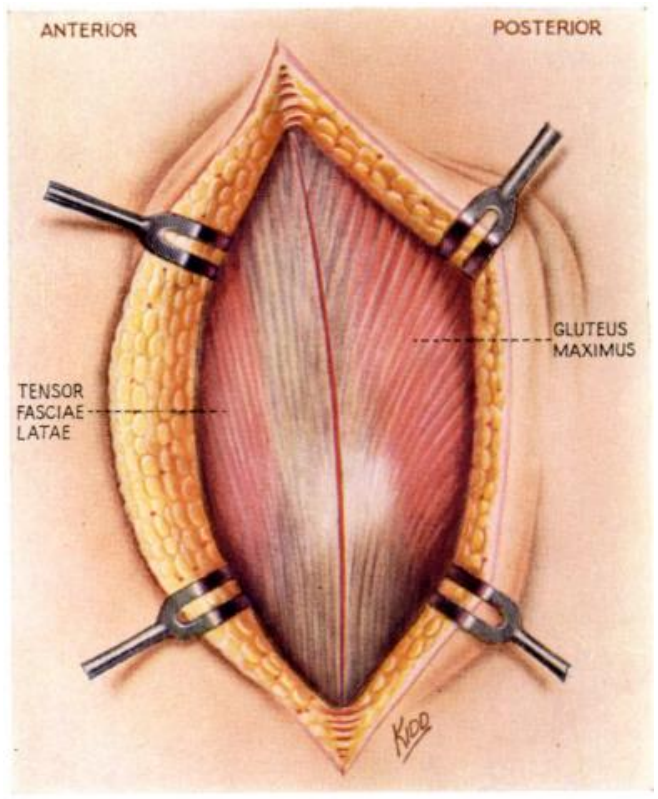

Fig. 2

Figure 1-skin incision. Figure 2-Incision of fascia lata between tensor fasciac latate in front and glutens maximus behind.

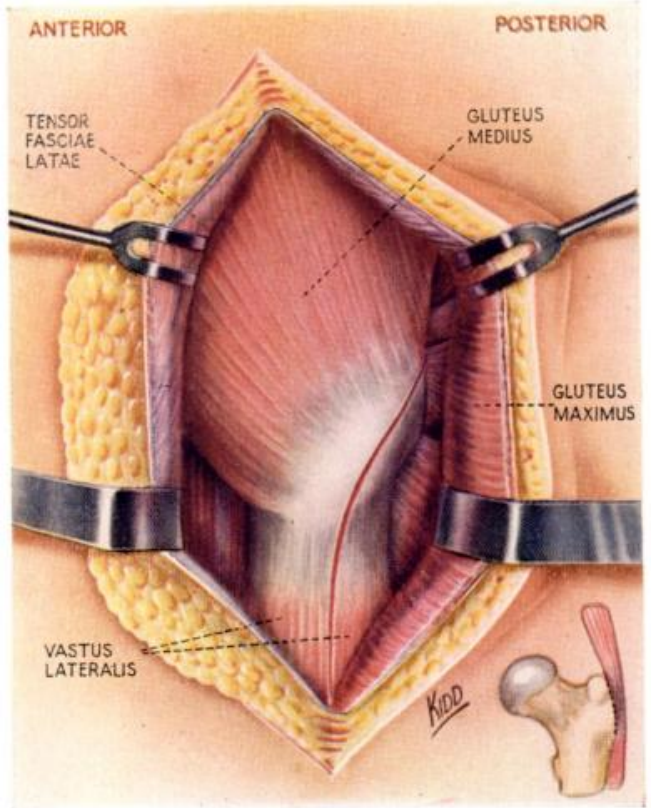

Fig. 3

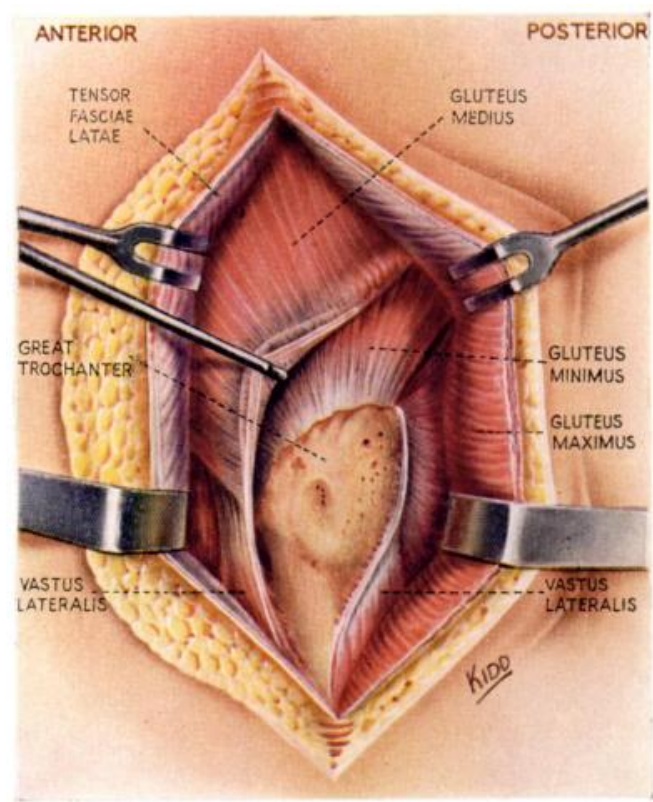

FIG. 4

Figure 3-Incision at posterior margin of gluteus medus and vastus lateralis, showing line at which loosening of periosteum of trochanter commences. Figure 4-Anterior displacement of gluteus medius and vastus lateralis ir. continuity.

VOl. 36 B, No. 3, AUgust 1954 
it satisfactorily with a raspatory. The portion of the vastus lateralis which is peeled off in this way includes, of course, that attached to the upper part of the linea aspera, the inferior border of the greater trochanter and part of the shaft of the femur. The whole combined muscle mass-gluteus medius and vastus lateralis with their tendinous junction-is then displaced forwards (Fig. 4). There is no difficulty in doing this, though it may be necessary to detach a small part of the vastus lateralis arising from the intertrochanteric line, and also to divide any anterior attachment that gluteus medius may have to minimus. The rest of the operation is similar to any other lateral or postero-lateral approach; the tendon of gluteus minimus is split and divided (Fig. 5) and retracted upwards to expose the capsule of the hip joint (Fig. 6).

At two points bleeding may occur-one from the ascending branch of the medial circumflex artety behind the greater trochanter and the other from the transverse branch of the lateral femoral circumflex artery deep to the vastus lateralis, but neither is troublesome.

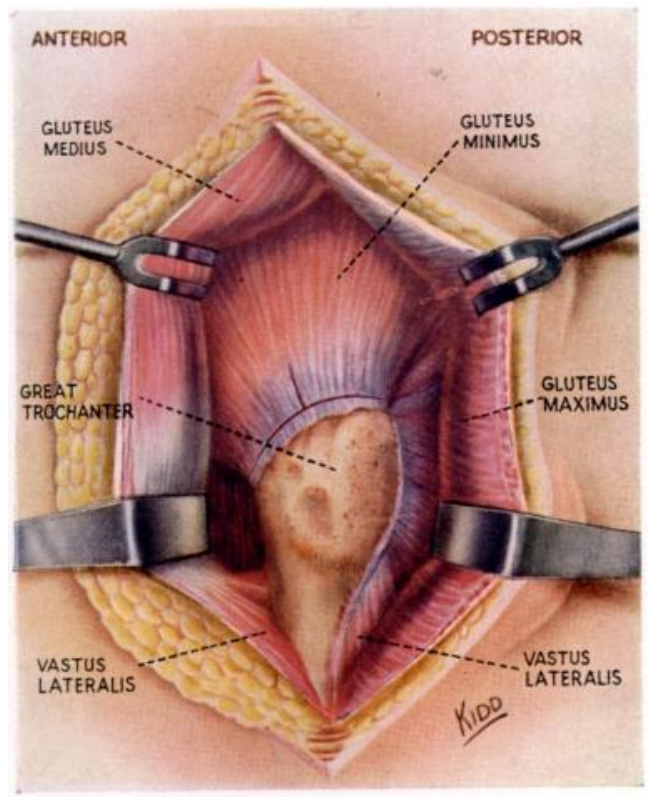

FIG. 5

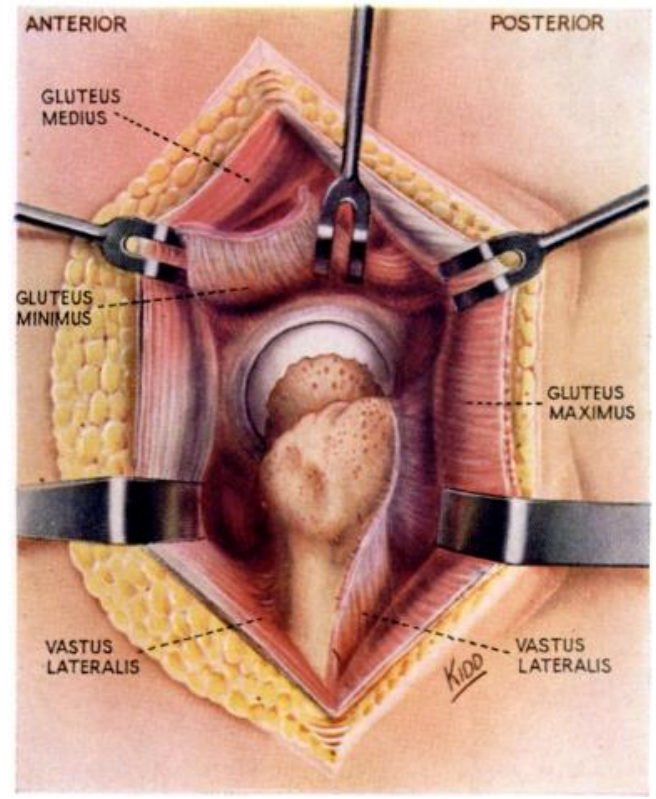

FIG. 6

Figure 5-Gluteus minimus revealed with line for division of its tendon. Figure 6-Hip joint revealed with neck and upper shaft of femur.

After finishing work on the hip joint, closure is carried out in layers. It is usual to suture the gluteus minimus and the capsule in one; the gluteus medius and vastus lateralis are returned to their original position and the tendinous part is sutured to the undisturbed part of vastus lateralis, to the deep insertion of gluteus maximus and to the upper part of the quadratus femoris. This point in the procedure is facilitated by having the leg abducted so that the tension on gluteus medius is reduced. About three strong sutures are necessary and chromic catgut is used.

\section{DISGUSSION}

Approaches to the hip joint fall, in principle, into two main groups. 1) Operations which approach the joint in front of, or behind, an intact gluteus medius (Hueter's and Langenbeck's incisions respectively). Access, however, is not easy and depends on very experienced 
retraction and " positioning " of the limb, and even then the view of the hip joint is somewhat restricted. 2) Operations in which the continuity of the gluteus medius is divided either by detaching part of its origin (Sprengl, Smith-Petersen), or by division of its insertion through the tendon or through the trochanter (Kocher, Murphy, Ollier, Brackett). Many surgeons prefer the wider and more ample exposure of this latter method. It is doubtful, however, whether a severed gluteus medius can subsequently be as strong as an undivided muscle, and the diversity of methods of reattaching the trochanteric insertion suggests the uncertainty of efficient union.

We believe that the manoeuvre of displacing gluteus medius introduces a third principle of approaching the hip joint in that the muscle, while still intact, is turned aside, and not divided either at its origin or insertion. Continuity is not interrupted and early restoration of abduction power has been secured.

\section{REFERENCES}

Gibson, A. (1950): Posterior Exposure of the Hip Joint. Journal of Bone and Joint Surgery, 32-B, 183. Kocher, T. (1903): Text-Book of Operative Surgery. London: Adam and Charles Black, 360.

LANGENBeck, B. v. (1874): Ueber die Schussverletzungen des Hüftgelenks. Archiv für Kilinische Chirurgie, 16, 294.

Mathied, P. (1937): Voies de Découverte de la Hanche. In Traité de Chirurgie Orthopédique, ed. L. Ombrédanne et P. Mathieu. Tome 4, 3,041, 3,043. Paris: Masson et Cie. (Heuter and Ollier incisions.)

Smith-Petersen, M. N. (1949): Approach to and Exposure of the Hip Joint for Mold Arthroplasty. Journal of Bone and Joint Surgery, 31-A, 40.

SPRFxGel, O. (1900): Zur operativen Nachbehandlung alter Hüftresectionen. Zeitschrift für Orthopädische Chirurgie, 7, 387. 